\title{
Decision-making for preradiation dental extractions is based primarily on experience and opinion
}

\section{What influences clinicians' preradiation dental extraction decisions when treating patients who have head and neck cancer?}

\author{
Bruins HH, Jolly DE, Koole R. Preradiation dental extraction \\ decisions in patients with head and neck cancer. Oral Surg Oral \\ Med Oral Pathol Oral Radiol Endod 1999; 88:406-412.
}

Design Questionnaire survey of 54 oral-maxillofacial surgeons and hospital-based dentists based in the USA, Europe and Australia. Results analysed using multiple regression.

Results The response rate was $81 \%$ (44 responses). Only nine clinicians (20\%) reported using printed clinical guidelines for preradiation dental screening. As cues for extraction decisions, dental conditions had a relative importance of $62 \%$, radiation dose $10 \%$ and tooth functionality $6 \%$. Certainty about whether their decision was appropriate was significantly influenced only by radiation dose. Some respondents reported that their decision-making was largely influenced by patients' previous dental performance and by socio-economic considerations, which were not included in the questionnaire. Correlation between the clinicians' policies and the model for dental decision support (MDDS) was high $(r=0.85)$.

Conclusions Clinicians' decision-making in this field appears to be based primarily on clinical experience and opinions rather than evidence-based clinical guidelines. These results need to be taken into consideration for further validation of the MDDS.

\section{Commentary}

Much attention has been focused on clinical-decision analysis in this field and the authors should be applauded for their efforts to develop and validate their decision model, MDDS, for preradiation extractions. ${ }^{1}$ The design of this case-based survey was clearly described, despite being simplistic in its ability to mirror closely real-life clinical situations, the expert population surveyed was appropriate, and it yielded high response rates.

The authors set out to validate their decision model by using the survey instrument to help define two objectives. In their first objective, "to study how variations in dental-related and radiotherapy-related risk factors influence clinicians' preradiation dental decision-making", the authors identified two dependent variables: whether or not to extract a tooth preradiation; and how certain the clinician is about the decision.

Six independent risk factor variables were identified, namely periodontal condition, endodontic condition, impacted teeth, radiation dose, location of the tooth and functionality of the tooth. Although it is clear that these variables were converted into dummy variables before running the multiple regression analysis, they were not specifically defined for the reader, making the results difficult to interpret.

Multiple linear regression was chosen to compare the effects of the independent variables on each of the dependent variables, yet

Address for correspondence: HH Bruins, Department of Oral and Maxillofacial Surgery, University Medical Centre Utrecht, University of Utrecht, Utrecht, The Netherlands. the extraction decision seems to be a binomial outcome-variable and, as such, multiple logistic regression might have generated different results. The risk factors studied accounted for $88 \%$ and $49 \%$ of the decisions to extract a tooth and the certainty of the decision, respectively. Furthermore, the decision was significantly influenced by all risk factors other than location of tooth (ie, maxilla versus mandible), although the certainty was significantly influenced only by radiotherapy-related risk factors. Interestingly, the dental condition (periodontal, endodontic and degree of impaction taken together) was relatively more important in the decision to extract than the other risk factors, and the consensus between clinicians was less for moderate-risk dental conditions than for those of low or high risk.

The second objective, "to examine the matching of clinician policies with those generated by the decision model", was clearly defined although the authors failed to describe how this correlation was performed in their methods, making the resultant high positive correlation $(r=0.85)$ difficult to interpret.

The authors' conclusion that the results support their "previous assumption that policies seem to be based primarily on clinical experience and opinions rather than on evidence-based clinical guidelines" is justified. This is not so much because of the results of the survey but more because presently there are no evidence-based clinical guidelines, at least none validated by prospective multicentre clinical trials. The impact of other important risk factors such as oral hygiene, financial issues and patient compliance as not addressed in the survey (some are assessed in the model), and may further influence the decision to extract teeth preradiation. The next step in validating the model could be an expert consensus survey using "paper cases" to incorporate real-life clinical scenarios, possibly using a Delphi approach, ${ }^{2}$ or a multicentre cohort study, which is in fact in progress.

\section{Practice point}

- Preradiation extraction decisions are based on clinical experience: more research is needed to help develop evidence-based guidelines for the future.

\section{A Ross Kerr}

New York University College of Dentistry, New York, USA

1. Bruins $\mathrm{HH}$, Koole $\mathrm{R}$, Jolly DE. Pretherapy dental decisions in patients with head and neck cancer. A proposed model for dental decision support. Oral Surg Oral Med Oral Pathol Oral Radiol Endod 1998. 86.256-267.

2. Cramer CK, Epstein JB, Sheps SB, Schechter MT, Busser JR. Modified Delphi survey for decision analysis for prophylaxis of post-radiation osteonecrosis. Oral Oncol 2002; 38:574-583.

Evidence-Based Dentistry (2003) 4, 11.

doi:10.1038/sj.ebd. 6400157 